\title{
Supersymmetric mirror models and dimensional evolution of spacetime
}

\author{
Wanpeng Tan买 \\ Department of Physics, Institute for Structure and Nuclear Astrophysics (ISNAP), \\ and Joint Institute for Nuclear Astrophysics - \\ Center for the Evolution of Elements (JINA-CEE), \\ University of Notre Dame, Notre Dame, Indiana 46556, USA
}

(Dated: August 25, 2020)

\begin{abstract}
A dynamic view is conjectured for not only the universe but also the underlying theories in contrast to the convectional pursuance of a single unification theory. As the 4-d spacetime evolves dimension by dimension via the spontaneous symmetry breaking mechanism, supersymmetric mirror models consistently emerge one by one at different energy scales and scenarios involving different sets of particle species and interactions. Starting from random Planck fluctuations, the time dimension and its arrow are born in the time inflation process as the gravitational strength is weakened under a 1-d model of a "timeron" scalar field. The "timeron" decay then starts the hot big bang and generates Majorana fermions and $U(1)$ gauge bosons in 2-d spacetime. The next spontaneous symmetry breaking results in two space inflaton fields leading to a double space inflation process and emergence of two decoupled sectors of ordinary and mirror particles. In fully extended 4-d spacetime, the supersymmetric standard model with mirror matter before the electroweak phase transition and the subsequent pseudo-supersymmetric model due to staged quark condensation as previously proposed are justified. A set of principles are postulated under this new framework. In particular, new understanding of the evolving supersymmetry and $Z_{2}$ or generalized mirror symmetry is presented.
\end{abstract}

\footnotetext{
* wtan@nd.edu
} 


\section{INTRODUCTION}

Searching evidence beyond the Standard Model (BSM) and building such types of theoretical models have been the main focus during the past decades in the studies of particle physics and cosmology. There are some well established observational evidences in cosmology such as dark energy, dark matter, and baryon asymmetry of the universe, etc, which point to the needs of new physics [1]. On the other hand, the discovery of neutrino oscillations [2, 3] and hence neutrinos with masses is probably the first direct BSM evidence from the point of view of particle physics.

Due to the known tension between quantum theory and general relativity, a lot of theoretical efforts go to the studies of quantum gravity represented by string theory, loop quantum gravity, and many other models. Although making the admiring mathematical progresses in these efforts, such unification theories have not shown solid connections to known physics and promising results to solve any of the outstanding puzzles. This indicates that we might still be missing or misunderstanding some key principles, especially the ones regarding symmetries.

One of the interesting ideas is about supersymmetry (SUSY) that relates bosons to fermions and it has been applied in string theory and many other studies [4]. However, SUSY is most often used to generate a new copy of known elementary particles. Such an idea seems to be in a crisis since the Large Hadron Collider has not found any such SUSY particles in the predicted energy range. Could such an issue be due to our incorrect understanding of SUSY? Nambu demonstrated a different SUSY principle as he called it quasi-SUSY [5]. He observed the matching of degrees of freedom (DoF) between fermions and bosons in many models including a special case considering only one generation of fermions in the Standard Model (SM). Such an idea definitely sheds new light on SUSY in building the extended Standard Model with mirror matter [6].

Another key insight is from the non-mainstream mirror matter theory. The idea of mirror symmetry was originated from the seminal work by Lee and Yang on parity violation [7]. Could the SM elementary particles have an almost identical mirror copy instead of a SUSYlike copy? It is conceivable that there exist two sectors of particles sharing the same gravity but governed by two separate gauge groups [8]. Some early works on mirror matter theory had discussed interesting perspectives in terms of mainly cosmology [9-11]. Other attempts 
to introduce feeble interactions between the two sectors might be going in the wrong way [12 14. Latest works [6, 15, 20] by keeping only the essence of mirror matter theory may indeed lead us to the new BSM physics we have all been looking for.

Instead of pursuing a single unification-type model, perhaps we should adopt a dynamic view of the universe and introduce an evolution of gauge, SUSY, and mirror symmetries along with spacetime leading to a hierarchy of supersymmetric mirror models at different energy scales in the early universe. Such a dynamic view of the universe has been hinted by recent studies, e.g., a conjecture that a meta-stable de Sitter universe like our universe with positive dark energy belongs to the swampland or is incompatible in string theory [21], and a proof that global symmetries can not be perfectly conserved under the general principles of quantum gravity 22. The best known dynamic approach resulting in phase transitions is the spontaneous symmetry breaking (SSB) mechanism which has been applied in many physics subfields including particle physics as the so-called Higgs mechanism and the underlying fermion condensation theory [6, 18, 23, 24].

The last important ingredient is more like a reminder for physicists. Topological effects could be what new physics will present to us as perturbation theory is not all physics. In quantum field theory, the best known non-perturbative transition "instanton" was introduced by 't Hooft [25], which represents the tunneling effects of topologically inequivalent vacuum configurations. Later on, topological solutions like "sphaleron" were calculated as saddle point solutions of gauge fields [26]. Similar topological transitions ("quarkiton") were recently proposed for staged quark condensation due to the flavor chiral $U(1)$ anomaly and SSB [6, 18. As a matter of fact, baryons like protons and neutrons can be considered soliton solutions in QCD. Neutrino oscillations are most likely topological transitions as well. Instead of searching perturbative effects as in most of dark matter and other BSM studies, we should probably focus our attention on the possible topological manifestation such as ordinarymirror neutral hadron oscillations (e.g., $n-n^{\prime}$ and $K^{0}-K^{0^{\prime}}$ ) [19].

In this paper, a self-consistent dynamical framework is introduced by naturally applying both SUSY and $Z_{2} /$ mirror symmetries in the early universe and adopting the SSB mechanism to describe the phase transitions along the evolution of spacetime dimensions and the hierarchy of underlying models at different energy scales. First, we start with the extended Standard Model with mirror matter near the electroweak phase transition scale based on the recent developments on mirror matter theory [6, 15] 20] in 4-d spacetime. Then we introduce 
the 1-d model for emergence of the time dimension and its arrow out of quantum Planck fluctuations. A set of principles are discussed before the 2-d spacetime model is presented for a double space inflation process and generation of two sectors of ordinary and mirror particles. In the end, we speculate on the possible ways to incorporate the gravitational effects in this framework.

\section{SUPERSYMMETRIC STANDARD MODEL WITH MIRROR MATTER}

At energies above the electroweak phase transition (EWPT) scale of $\sim 10^{2} \mathrm{GeV}$, we extend the SM with a new gauge symmetry group of $U_{f}(6) \times S U_{c}(3) \times S U_{w}(2) \times U_{Y}(1)$ and a new sector of mirror particles under a similar mirror gauge group of $U_{f}(6)^{\prime} \times S U_{c}(3)^{\prime} \times S U_{w}(2)^{\prime} \times$ $U_{Y}(1)^{\prime}$ as studied in Refs. [6, 18]. Here $U_{f}(6)$ stands for the gauged flavor symmetry while the rest represent the symmetries of the well-studied SM gauge interactions. The Lagrangian of the ordinary sector can then be written as,

$$
\mathcal{L}_{\mathrm{O}}=-\frac{1}{4} G_{\mu \nu}^{a} G^{a \mu \nu}+i \bar{\psi}_{j} \gamma^{\mu} D_{\mu} \psi_{j}
$$

where $G_{\mu \nu}^{a}(a=1,2, \ldots, 48)$ is the gauge field strength tensor and the gauge covariant deriva-

tive $D_{\mu}=\partial_{\mu}-i g T^{a} A_{\mu}^{a}$ depends on gauge symmetry generators $T^{a}$ and gauge bosons $A_{\mu}^{a}$. The gauge coupling constant $g$ may be unified to be one initially at $\sim 10^{16} \mathrm{GeV}$ and then evolves differently for different subgroups at lower energies. The massless Dirac fermion fields $\psi_{j}$ include three generations of all quarks and leptons and can be rewritten as 48 left and right Weyl fermions with all chiral symmetries observed.

It is clear to see that the degrees of freedom (DoF) for gauge bosons and fermions are matched as $n_{f}=n_{b}=96$ for the ordinary sector [6] so that they can be in the same SUSY multiplet $\left(1, \frac{1}{2}\right)$. Eq. (1) as the on-shell Lagrangian therefore follows the unbroken $N=1$ supersymmetric Yang-Mills theory, i.e., invariant under the $N=1$ gauge SUSY transformations.

The mirror sector is essentially identical but completely decoupled from the ordinary sector as far as gauge interactions are concerned. Besides sharing the same gravity, i.e., the stage of spacetime, the two sectors are related under the mirror transformation $\mathcal{M}$ as follows,

$$
\mathcal{M}: \psi_{L} \rightarrow-\psi_{L}^{\prime}, \psi_{R} \rightarrow \psi_{R}^{\prime}, A_{\mu} \rightarrow A_{\mu}^{\prime}
$$


which notably performs differently by a negative sign for left- and right-handed fermion fields [6]. The corresponding mirror Lagrangian is essentially the same as Eq. (1) but for mirror particles and mirror gauge group. Note that it is the mirror symmetry, instead of SUSY as often assumed, that requires a new copy of particles in a new sector.

Now the question is how this model undergoes spontaneous symmetry breaking at EWPT. As proposed in Ref. [6, 18], some kind of pseudo-SUSY is preserved and staged quark condensation occurs at energies between $10^{2} \mathrm{GeV}-10^{2} \mathrm{MeV}$ along with SSB of the mirror symmetry and $U_{f}(6)$ as evidenced by the hierarchical quark and lepton masses. The Lagrangian of the ordinary sector becomes

$$
\begin{aligned}
\mathcal{L}_{\mathrm{O}} & =\mathcal{L}_{\left(1, \frac{1}{2}\right)}+\mathcal{L}_{\left(\frac{1}{2}, 0\right)}+\mathcal{L}_{\text {Higgs }} \\
\mathcal{L}_{\left(1, \frac{1}{2}\right)} & =-\frac{1}{4} G_{\mu \nu}^{a} G^{a \mu \nu}+i \bar{\psi}_{j}^{L} \gamma^{\mu} D_{\mu}^{L} \psi_{j}^{L}+i \bar{\psi}_{j}^{R} \gamma^{\mu} D_{\mu}^{R} \psi_{j}^{R} \\
\mathcal{L}_{\left(\frac{1}{2}, 0\right)} & =i \bar{\nu}_{n}^{R} \gamma^{\mu} \partial_{\mu} \nu_{n}^{R}+\frac{1}{2}\left(\partial_{\mu} \phi_{f}\right)\left(\partial^{\mu} \phi_{f}\right) \\
\mathcal{L}_{\text {Higgs }} & =-\sum_{j} y_{j}\left(\bar{\psi}_{j}^{L} \psi_{j}^{R} \phi_{f}+\text { h.c. }\right)+\sum_{f} \frac{1}{2} m_{f}^{2} \phi_{f}^{2}-\sum_{f} \frac{1}{8}\left(\phi_{f}\right)^{4}
\end{aligned}
$$

where $\mathcal{L}_{\left(1, \frac{1}{2}\right)}$ preserves an $N=1$ gauge pseudo-SUSY $\left(1, \frac{1}{2}\right)$ multiplet, $\mathcal{L}_{\left(\frac{1}{2}, 0\right)}$ presents three copies of chiral SUSY $\left(\frac{1}{2}, 0\right)$ multiplets, and $\mathcal{L}_{\text {Higgs }}$ provides the mass and Higgs potential terms via the Higgs mechanism. Here the gauge group is reduced to $S U_{c}(3) \times S U_{L}(2) \times U_{Y}(1)$ with $S U_{L}(2)$ applying only to left-handed fermions. To keep both gauge bosons and fermions in the same $\left(1, \frac{1}{2}\right)$ multiplet, the matching of DoFs is realized with $n_{f}=n_{b}=90$ as pseudoNambu-Goldstone bosons (pNGB) from the $U_{f}(6)$ symmetry breaking provide 63 DoFs and neutrino degeneracy (i.e., both sectors share the same set of neutrinos and only left-handed neutrinos participating in gauge interactions of the ordinary sector) reduces DoFs of gaugeparticipating fermions by six [6]. The three right-handed neutrinos $\left(\nu_{n}^{R}, n=1,2,3\right)$ form the three chiral SUSY multiplets with the six real Higgs scalars $\left(\phi_{f} \sim\left\langle\bar{q}_{f} q_{f}\right\rangle, f=1, \ldots, 6\right)$ from staged quark condensation [6, 18]. The non-vanishing vacuum expectation values of these composite Higgs scalars lead to the non-zero mass terms of all fermions that explicitly break SUSY into pseudo-SUSY. Note that the tree level coefficient of the Higgs quartic terms are fixed to $1 / 8$ using the maximally interacting principle [6] and will be discussed later. Therefore, overall the ordinary sector after the symmetry breaking shows a maximal $N=4$ pseudo-SUSY.

The mirror sector basically behaves the same obeying a similar $N=4$ pseudo-SUSY 
except that in this case the mirror gauge group is reduced to $S U_{c}(3)^{\prime} \times S U_{R}(2)^{\prime} \times U_{Y}(1)^{\prime}$ and the left-handed neutrinos have decoupled from the mirror gauge interactions forming the three chiral SUSY multiplets with mirror Higgs fields from mirror quark condensation. The mirror symmetry transformation $\mathcal{M}$ is then, after considering the scalar fields, as follows,

$$
\mathcal{M}: \psi_{L} \rightarrow-\psi_{L}^{\prime}, \psi_{R} \rightarrow \psi_{R}^{\prime}, A_{\mu} \rightarrow A_{\mu}^{\prime}, \phi \rightarrow-\phi^{\prime}
$$

Neutrinos play a critical role since they are shared by the two sectors causing the degeneracy. In particular, their mass terms have to be constructed from the combination of the Yukawa terms of both sectors as follows [6],

$$
-y\left(\bar{\nu}_{L} \nu_{R} \phi+\bar{\nu}_{L}^{\prime} \nu_{R}^{\prime} \phi^{\prime}+\text { h.c. }\right)=-y\left(\bar{\nu}_{L} \nu_{R}\left(\phi-\phi^{\prime}\right)+\text { h.c. }\right)
$$

where the neutrino degeneracy relations of $\nu_{L}=-\nu_{L}^{\prime}$ and $\nu_{R}=\nu_{R}^{\prime}$ are applied. It is easy to see that masses of the neutrinos are determined by the ordinary-mirror mass splitting scale of $\left\langle\phi-\phi^{\prime}\right\rangle \sim \delta v$ with fairly well constrained relative scale of $\delta v / v=10^{-15}-10^{-14}$ [6, 15] 19]. Note that neutrinos are Dirac fermions and therefore no neutrinoless double beta decay is possible.

The above formalism gives an immediate extension to SM that preserves all the good results of SM. More intriguingly, supersymmetry is implicitly embedded in the extended model. The known gauge bosons and elementary fermions are actually pseudo-SUSY partners to each other. The missing DoFs in the SUSY boson-fermion balance are provided by the pNGBs from staged quark condensation of the $U_{f}(6)$ breakdown. It is the mirror symmetry that requires an almost identical copy of elementary particles in an almost completely decoupled sector.

Such a supersymmetric standard model with mirror matter extension can provide explanations for puzzles like the above-mentioned nature and masses of neutrinos and observed dark energy scale of $10^{-3} \mathrm{eV}$ assuming that gravitational vacuum energy is determined by a coherent sum of all scalar fields [6]. Along with predicted neutral hadron-mirror hadron oscillations, in particular, the two most important cases of $n-n^{\prime}$ and $K^{0}-K^{0^{\prime}}$ for neutrons and kaons, we can quantitatively and consistently explain many other enigmas such as neutron lifetime anomaly and dark matter [15], baryon asymmetry of the universe [18], nucleosynthesis and evolution of stars [16], ultra-high energy cosmic rays [17], and unitarity of the CKM matrix [19]. More importantly, new testable predictions and feasible experiments 
have been proposed and are relatively easy to carry out to directly verify or refute this new model [19].

Now the question is on the origin of the gauge group $U_{f}(6) \times S U_{c}(3) \times S U_{w}(2) \times U_{Y}(1)$ and its mirror counterpart. A naive guess would be that it should be related to the underlying spacetime and SUSY. Evidently, our base manifold (extended 4-d spacetime) defines gravity while the internal/local fiber space (e.g., as tangent space of 4-d spacetime and/or compacified Calabi-Yau manifold) defines the gauge group. The holonomy group of the 4-d Riemannian spacetime is $O(1,3) \sim S O(1,3) \times Z_{2}$ where $Z_{2}$ is the mirror symmetry and the Lorentz group is locally isomorphic to the double $S U(2)$ gauge group,

$$
S O(1,3) \sim S U(2) \times S U(2)^{\prime}
$$

which can act on the tangent space and provide the $S U_{w}(2)$ gauge for both sectors. Its maximal torus subgroup (roughly, maximal abelian Lie subgroup) is just the so-called Clifford torus $U(1) \times U(1)^{\prime}$ where the two circles are embedded independently in their own 2-d space of the 4-d tangent space. This could be considered as the origin of gauge $U_{Y}(1)$ and $U_{Y}^{\prime}(1)$ for the two independent sectors, respectively. Therefore, both $U_{Y}(1)$ and $S U_{w}(2)$ are originated directly from 4-d spacetime which is evidently the stage for all fermion particles.

Supersymmetric string theory requires the existence of an additional 6-d Calabi-Yau manifold to be consistent [27]. The holonomy group $S U(3)$ of such a manifold could provide the color gauge $S U_{c}(3)$. In particular, the well-known mirror symmetry of Calabi-Yau manifolds [28] could be related to the mirror symmetry of this work, i.e., one 6-d Calabi-Yau manifold is for the ordinary sector while the mirror Calabi-Yau manifold is for the mirror sector. Because this 6-d manifold is separate from 4-d spacetime, it naturally requires a new set of fermions, i.e., quarks for $S U_{c}(3)$.

Now we can see how the flavor gauge $U_{f}(6)$ is generated. $S U_{w}(2)$ stems from 4-d spacetime and universally ensures fermion doublets. However, $S U_{c}(3)$ is originated from the compacified Calabi-Yau manifold and therefore applies only to quarks. The different origins of $S U_{w}(2)$ and $S U_{c}(3)$ ask for two set of fermions, i.e., quarks and leptons. Since both $U_{Y}(1)$ and $S U_{w}(2)$ come from extended 4-d spacetime so that they apply to all fermions, i.e., both quarks and leptons. As easily calculated, the SUSY principle, i.e., matching of boson-fermion DoFs $\left(n_{b}=n_{f}\right)$ requires that the number of fermion generations be either $n=1$ or $n=3$, given the gauge group of $U_{f}(2 n) \times S U_{c}(3) \times S U_{w}(2) \times U_{Y}(1)$. Nature 
selects $n=3$ leading to maximal supersymmetry, i.e., $N=4$ pseudo-SUSY after quark condensation. The $U_{f}(6)$ gauge could be just the holonomy group of the Kähler fiber space induced by six flavor fermions (i.e., three generations of $S U_{w}(2)$ doublets).

One possible explanation for three generations could be that there are three independent ways to generate the maximal torus group of $U(1) \times U(1)^{\prime}$ out of 4 -d spacetime. Using the simple $n_{b}=n_{f}$ SUSY principle, we can also derive a simple constraint on the Calabi-Yau manifold assuming three generation fermions. Considering that the holonomy group of a 2k-dimensional Calabi-Yau manifold is $S U(k)$, we can easily obtain $k=3$ or 9 simply by requiring $n_{b}=n_{f}$.

\section{DIMENSIONAL EVOLUTION OF SPACETIME AND THE ARROW OF TIME}

It is imaginable that the universe has a quantum origin at Planck scales. Here we assume that spacetime starts with four dimensions, each of which is at its baby size, i.e., the Planck scale. Then the 1-d time starts to grow into its current extended time dimension. In 1-d time, only a true real scalar field $\phi=\phi(t)$ can exist. The Lagrangian can be written simply as,

$$
\mathcal{L}=\frac{1}{2} \dot{\phi}^{2}
$$

for a massless $\phi$ field. Such a 1-d Riemannian manifold has no intrinsic curvature and therefore the action can be written as,

$$
\mathcal{S}=\frac{1}{M_{p}^{3}} \int d t \mathcal{L}
$$

where the factor involving the Planck mass $M_{p}$ is obtained by a trivial integration over the three unextended or uninflated space dimensions.

The holonomy group of a 1-d Riemannian manifold is $O(1)=Z_{2}$. As shown below, this is actually the time reversal symmetry and also a primordial hint for other $Z_{2}$ symmetries and their breakdowns at later stages of the universe. To break this time reversal $Z_{2}$ symmetry, we can apply the SSB mechanism by introducing a potential term $V(\phi)=-m^{2} \phi^{2} / 2+\phi^{4} / 8$ for the scalar $\phi$ to acquire mass and then the SSB Lagrangian becomes

$$
\mathcal{L}=\frac{1}{2} \dot{\phi}^{2}+\frac{1}{2} m^{2} \phi^{2}-\frac{1}{8} \phi^{4}
$$




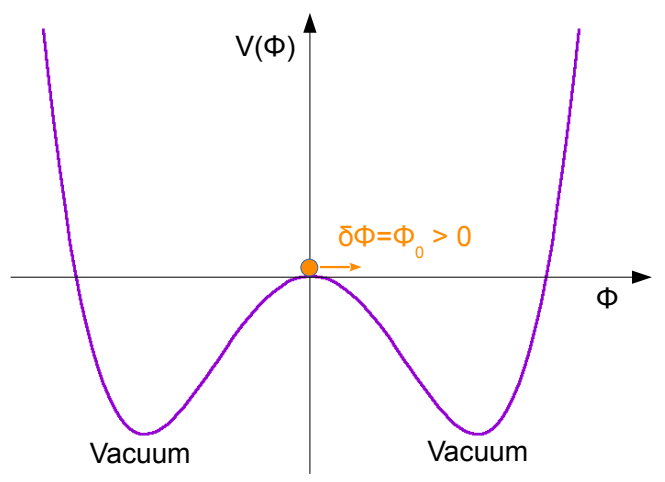

FIG. 1. The potential of the scalar "timeron" field $\phi$ is shown to demonstrate the time inflation and the birth of the time arrow.

where the quartic term and the factor of $1 / 8$ are obtained from the mass or quadratic term using the maximally interacting principle [6] as discussed later.

Before the SSB, the sole kinetic term as in Eq. (10) represents eternal quantum fluctuations. After the SSB with Eq. (12), the time reversal symmetry is broken as the scalar $\phi$ rolling down its potential towards one of the two emerging true vacuum configurations. The chosen direction of the rolling defines the arrow of the time as shown in Fig. (1). The equation of motion easily obtained from Eq. (12) can be written as,

$$
\ddot{\phi}=m^{2} \phi-\frac{1}{2} \phi^{3} \text {. }
$$

Assuming that the initial push $\delta \phi=\phi_{0}$ is much smaller than $\phi$ 's mass $m$, the 2nd term in Eq. (13) is negligible and hence the solution showing an inflationary behavior is simply,

$$
\phi(t)=\phi_{0} \exp (m t)
$$

where the time arrow is chosen towards the positive field direction without loss of generality as shown in Fig. (1). This provides the time-like inflation to make time smooth and causal. Therefore, the scalar field $\phi$ serves as a time inflaton or "timeron" to start the timer of the universe. Original fluctuations at the Planck scale are so chaotic that the original baby-sized spacetime is by no means smooth or causal. But this time inflation process can ensure a causally smooth time dimension afterwards.

During the time inflation process, the vacuum or potential entropy can be defined as $S_{\text {vac }}=E / T$ where the timeron field energy $E \propto \phi^{2} \propto \exp (2 m t)$ and the temperature drops exponentially as $T=\exp (-m t)$. Therefore, the vacuum entropy increases enormously as 


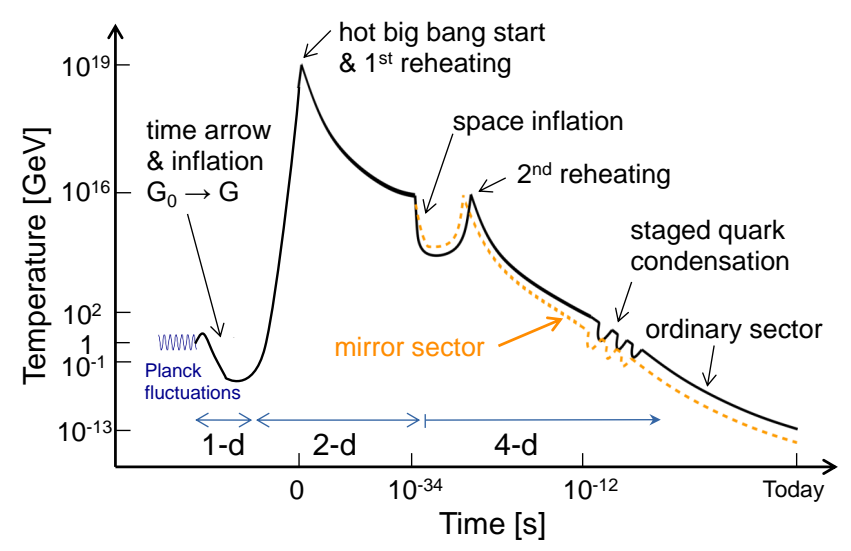

FIG. 2. The schematic diagram (not to scale) is shown for the dynamic history of the universe where the temperature is mostly determined by massless particles and the dips reflect various stages of spontaneous symmetry breaking and emergence of new energy scales leading to each new cosmic era. See text for details.

$S_{\text {vac }} \propto \exp (3 m t)$ and this makes the arrow of time also aligning with the thermodynamic arrow as required by the second law of thermodynamics. In the subsequent timeron decay or reheating process, the vacuum entropy will then be converted into the particle entropy.

One likely way to ensure $\delta \phi=\phi_{0}<<m$ is by considering that the strength of the gravitational force may be restored to its maximum in the beginning, in other words, the original gravitational constant $G_{0}$ may be $10^{38}$ times larger than its current value $G$. It is conceivable that the gravity may get weakened as spacetime is exponentially extended. This leads to much smaller Planck mass in Eq. (11) $M_{p} \sim 1 / \sqrt{G_{0}} \sim 1 \mathrm{GeV}$ and therefore the initial temperature or fluctuations in spacetime and "timeron" $\phi$ at the very beginning are also on the same scale with the initial push $\delta \phi=\phi_{0}$ served from these random fluctuations. On the other hand, the emerging mass of $\phi$ should be $m \sim 1 / \sqrt{G} \sim 10^{19} \mathrm{GeV}$, i.e., the Planck mass corresponding to the weakened gravity due to inflated spacetime. At the end of the time inflation (i.e., settled in the new vacuum), "timeron" $\phi$ will decay into new particles that will be discussed later and start the hot big bang at $\sim 10^{19} \mathrm{GeV}$ as shown in Fig. (2).

\section{THE FEYNMAN AND SUSY MIRROR PRINCIPLES}

Before we construct other models at different spacetime dimensions to bridge the dynamic evolution of the universe from the birth of time to the SM physics, we will sort out a set 
of principles [6] under the Feynman path integration formalism for constructing the proper Lagrangian and action for a given system. Under this framework, the probability amplitude of a physical system can be obtained as a coherent sum of all possible configurations weighted by a phase factor of the action of each configuration,

$$
A=\sum_{\text {configurations }} \exp (i \mathcal{S} / \hbar)
$$

where the action is determined by an integration of Lagrangian over D-dimensional flat spacetime,

$$
\mathcal{S}=\int d^{D} x \mathcal{L}
$$

Each configuration is associated with its own action or Lagrangian and all configurations can contribute to the probability amplitude. But most of them tend to vanish or cancel out each other. Here we postulate the principles for the action and Lagrangian of the most probable configuration:

1. It has to be maximally symmetric.

2. It has to be finite or renormalizable.

3. It has to be complete to include all possible terms.

4. Spontaneous symmetry breaking (SSB) provides the dynamic mechanism.

These principles ensure that the Lagrangian should include all possible renormalizable maximal-symmetry-obeying dimension- $D$ (or dimension-4 for 4-d spacetime) terms. At least some of these have been commonly applied in constructing Lagrangians for many physical systems. Finiteness essentially selects renormalizable dimension-4 terms as nonrenormalizable terms will cause infinity in the action making its contribution to the amplitude vanish. In a given system like our universe, the maximal symmetry postulate requires that all the terms in Lagrangian have to obey Lorentz (for base manifold - extended spacetime), gauge (for compacified and fiber spaces), and other (e.g., SUSY and mirror) symmetries. It has to be maximally symmetric as any asymmetric constructions of Lagrangian tend to cancel out with corresponding asymmetric counterparts. Last but not least, SSB is essential to provide the phase transition dynamics for the emergence of hierarchical supersymmetric mirror models. 
However, an inferred principle (i.e., the " maximally interacting" principle named in Ref. [6]) needs extra attention. If a term $V$ is in the Lagrangian and $V^{2} /\left(2 m^{D}\right)$ is renormalizable (where the mass scale factor of $1 / m^{D}$ is to make it a dimension- $D$ term), then it has to be in the Lagrangian as well. This has its special application in SSB when a new energy scale emerges. For example, in the extended standard model with mirror matter [6], the quartic Higgs term due to quark condensation has a fixed factor of $1 / 8$ as in Eq. (6) according to this principle.

We can also see how it works in the above-discussed 1-d time model. A general invariant renormalizable potential of the true scalar field can be written as $V(\phi)=\lambda_{1} m^{3} \phi+\lambda_{2} m^{2} \phi^{2}+$ $\lambda_{3} m \phi^{3}+\lambda_{4} \phi^{4}$. We can set $\lambda_{1}=0$ as this term is just a trivial shift of $\phi$ and $\lambda_{2}=-1 / 2$ for $m$ to represent $\phi$ 's mass. The cubic term defines the asymmetry of the two vacuum configurations and the two asymmetric cases of $\pm \lambda_{3}$ will cancel out each other. According to the maximal symmetry principle, we can then set $\lambda_{3}=0$ for more symmetric vacuum degeneracy. The last quartic term can be constructed from the quadratic term using the maximally interacting principle to have the factor of $\lambda_{4}=1 / 8$ leading to Eq. (12).

The SUSY principle states that a model has to be (pseudo-)supersymmetric and includes only SUSY-allowed fields. For extended superspace $\left(x^{\mu}, \theta^{\alpha}, \bar{\theta}^{\dot{\beta}}\right)$ where $x^{\mu}$ are spacetime coordinates and $\theta^{\alpha}$ and $\bar{\theta}^{\dot{\beta}}$ are corresponding anticommuting Grassmannian coordinates, a general scalar function $F\left(x^{\mu}, \theta^{\alpha}, \bar{\theta}^{\dot{\beta}}\right)$ can be expanded in finite number of terms up to $\theta^{2} \bar{\theta}^{2}$ in 4-d spacetime [4] leading to the above-discussed 4-d SUSY and pseudo-SUSY models of Eqs. (1 9). In 1-d time, $\theta$ and $\bar{\theta}$ do not exist so that the general function $F=\phi(t)$ is trivial, i.e., only a scalar field can exist as presented in the 1-d models of Eqs. 10 12). In 2-d spacetime, $F=\phi(x, t)+\theta \psi(x, t)+\bar{\theta} \bar{\psi}(x, t)+\theta \sigma_{\mu} \bar{\theta} A^{\mu}(x, t)$ where more fields like Majorana spinor $\psi$ and gauge boson $A^{\mu}$ are allowed and will be discussed below. In particular, under varied spacetime dimensions, the scalar fields provide the mechanism of SSB and new mass scales for the dynamic evolution of the universe. In light of the other principles especially the principle of finiteness, SUSY might also help constrain $D \leq 4$ for our spacetime.

The $Z_{2}$ or the generalized mirror symmetry in the holonomy group $O(n) \sim S O(n) \times Z_{2}$ of spacetime and its spontaneous breaking play a critical role at different dimensions. In 1-d time, it is the time-reversal or time-mirror symmetry. In 4-d spacetime, it is the mattermirror symmetry between the two sectors. In 2-d spacetime as discussed next, it is the chiral-mirror symmetry. 


\section{HIERARCHY OF SUPERSYMMETRIC MIRROR MODELS}

Now we can consider further evolution of spacetime after the time arrow and inflated time dimension are born. That is, 1-d space also starts to grow from its baby size. In 2-d spacetime, the holonomy group is $O(1,1) \sim U(1) \times Z_{2}$ and as we will see, the first gauge group, SUSY, and Majorana fermions are born at this stage. We can easily write down the Lagrangian and the action at this stage,

$$
\begin{aligned}
\mathcal{L} & =-\frac{1}{4} F_{\mu \nu} F^{\mu \nu}+\frac{i}{2} \lambda^{\dagger} \bar{\sigma}^{\mu} \partial_{\mu} \lambda+\text { h.c. } \\
\mathcal{S} & =\frac{1}{M_{p}^{2}} \int d t d x \mathcal{L}
\end{aligned}
$$

where the $U(1)$ gauge field strength $F_{\mu \nu}=\partial_{\mu} A_{\nu}-\partial_{\nu} A_{\mu}$ and the Majorana fermion $\lambda$ has to be neutral and does not couple to the gauge field $A_{\mu}$. Both the Majorana fermion and the gauge boson are massless and have two components or DoFs. They form the simplest $N=1$ abelian gauge SUSY multiplet $(1,1 / 2)$ with the on-shell Lagrangian of Eq. (17). At the end of the time inflation, the true scalar "timeron" $\phi$ with energy of about $10^{19} \mathrm{GeV}$ will decay into these two particles when the 2-d spacetime emerges. The $Z_{2}$ symmetry is just the chiral symmetry between left- and right-handed Majorana fermions at this stage.

Again, SSB will occur when Majorana fermions start to condense into a couple of pseudoscalars of $\phi$ and $\phi^{\prime}$. The $U(1)$ gauge symmetry is broken and the new Lagrangian at the new emerging energy scale of $10^{16} \mathrm{GeV}$ is composed of two parts,

$$
\begin{aligned}
\mathcal{L}_{\left(\frac{1}{2}, 0\right)} & =\frac{1}{2}\left(\partial_{\mu} \phi \partial^{\mu} \phi+\partial_{\mu} \phi^{\prime} \partial^{\mu} \phi^{\prime}\right)+\frac{i}{2} \lambda^{\dagger} \bar{\sigma}^{\mu} \partial_{\mu} \lambda+h . c . \\
\mathcal{L}_{\text {Higgs }} & =-\left(\lambda_{L}^{\dagger} \lambda_{R}\left(\phi+i \phi^{\prime}\right)+\text { h.c. }\right)+\frac{1}{2} m^{2} \phi^{2}-\frac{1}{8} \phi^{4}+\frac{1}{2} m^{\prime 2} \phi^{\prime 2}-\frac{1}{8}{\phi^{\prime}}^{4}
\end{aligned}
$$

where the first part shows the symmetry of $N=1$ pseudo-SUSY multiplet $\left(\frac{1}{2}, 0\right)$ and the second part presents a Higgs-like mechanism with both scalars acquiring masses $\left(m \neq m^{\prime}\right)$ of about $10^{16} \mathrm{GeV}$. If we assume that the mass splitting parameter $\delta m / m \propto T$ and it is of order unity initially at temperature $T \sim 10^{16} \mathrm{GeV}$, then the temperature difference between the two sectors is reasonably understood and the tiny mass splitting parameter of about $10^{-14}$ [15] for later quark condensation at $T \sim 10^{2} \mathrm{GeV}$ is also explained.

The two scalar fields of $\phi$ and $\phi^{\prime}$ then start to drive the inflation as two more space dimensions are extended. At the end of the space inflation, we have fully inflated the 4-d spacetime. Meanwhile the new gauge symmetry of $U_{f}(6) \times S U_{c}(3) \times S U_{w}(2) \times U_{Y}(1)$ and the 
corresponding $N=1$ gauge SUSY as discussed earlier emerge for new particles including massless quarks and leptons. The space inflatons $\phi$ and $\phi^{\prime}$ then decay into new particles to reheat the universe and form the two ordinary and mirror sectors, respectively. The different energy scales of the two inflatons causes different temperatures $\left(T^{\prime}<T\right)$ during reheating in the two sectors that will satisfy the constraints of big bang nucleosynthesis [10, 11]. As shown in Eqs. (1 12 , the $Z_{2}$ symmetry is the mirror symmetry at this stage with fully developed 4-d spacetime. As discussed earlier, the mirror symmetry and SUSY then undergo spontaneous symmetry breaking due to staged quark condensation starting at $\sim 10^{2} \mathrm{GeV}$ and eventually lead to the $N=4$ pseudo-SUSY theory described by Eqs. (3-6 6 ) that very much resemble the Standard Model but with a mirror extension. A schematic diagram of the dynamic evolution of the universe and spacetime dimensions is shown in Fig. (2).

One important prediction from this inflation model is that the space dimensions undergo a double-inflation process and hence can present a signature of a dipole mode. This might explain the recent discovery of a large dipole component of cosmic acceleration in a reanalysis of type Ia supernova data by Colin et al. [29].

\section{OUTLOOK AND FURTHER DISCUSSIONS}

Such a dynamic view for not only the universe but also the underlying theoretical models seriously challenges the conventional wisdom of a single fundamental unification model for the universe. Nature seems to tell us that fundamentally different physics may emerge at different energy scales or scenarios to which we have to apply different theories. As proposed in this work, the emergence of hierarchical supersymmeric mirror models at different scales is directly driven by the dynamic evolution of spacetime dimensions.

However, more details need to be answered. For example, could we obtain the different energy scales such as $10^{19}, 10^{16}$, and $10^{2} \mathrm{GeV}$ from some first principles other than the anthropic principle? What is exactly the dynamics of spacetime? Would the last two space dimensions emerge sequentially or simutaneously? How should gravity be incorporated with other particle fields?

A naive inclusion of gravity could be $\mathcal{S}=\int d^{4} x \sqrt{-g}\left(-\frac{1}{16 \pi G} R+\mathcal{L}_{m}+\mathcal{L}_{\phi}\right)$ in 4-d spacetime and $\mathcal{S}=\int d^{2} x \sqrt{-g} G\left(-\frac{1}{16 \pi G} R+\mathcal{L}_{m}+\mathcal{L}_{\phi}\right)$ in 2-d spacetime with two uninflated dimensions 
integrated as $G$, where $R$ is the Ricci curvature scalar, $\mathcal{L}_{m}$ is the Lagrangian of kinetic and mass terms of all fields, and $\mathcal{L}_{\phi}$ is the quartic terms of scalar fields. It is trivially reduced to the simple results of Eqs. 10,12 in the 1-d case where the curvature vanishes.

Clearly, $\mathcal{L}_{m}$ contributes to the energy-momentum tensor of the gravity theory (e.g., in the Einstein's equations of 4-d spacetime). For the case of a single scalar field $\phi$, it gives a nonvanishing cosmological constant as $\Lambda=\pi G\langle\phi\rangle^{4}$. However, for more complicated cases like the supersymmetric mirror model of Eqs. (377), we have to distinguish the three different vacuum configurations for ordinary and mirror gauge interactions and gravity, respectively. In particular, the vacuum energy for gravity should be determined by the coherent sum of all scalar fields (even from different gauge sectors) since they share the same gravity. As such, the gravitational vacuum energy density is $\rho_{\Lambda}=\frac{1}{8}\left\langle\sum_{j} \phi_{j}\right\rangle^{4}$, which can explain the observed dark energy density of $\left(10^{-3} \mathrm{eV}\right)^{4}$ under our new model [6].

An immediate idea following this line of thought is that gravity may be emergent from the mean field effects of quantum fluctuations of spacetime after its inflation [30]. General relativity can then be treated as a mean field theory obtained from the mean field action,

$$
\mathcal{S}_{\text {grav }}=\int d^{4} x \sqrt{-g}\left(-\frac{1}{16 \pi G} R+\left\langle\mathcal{L}_{m}\right\rangle+\left\langle\mathcal{L}_{\phi}\right\rangle\right)
$$

where $\left\langle\mathcal{L}_{\phi}\right\rangle$ requires the coherent sum of all scalar fields for the proper cosmological constant. Under this scenario, gravity just defines the spacetime metric $g^{\mu \nu}$ as the smooth mean geometrical background for quantum particles [30]. But could we understand this better?

The idea from string theory may help. The 2-d worldsheet in string theory may be a good description of the complex structures in the gauge group. It can therefore provide

a complementary view from internal string structures of particle fields. For example, for extended 2-d spacetime, it could be regarded as a view from the other two unextended dimensions. For extended 4-d spacetime, it may be a view from the intrinsic complex nature of the compacified and fiber spaces. This may shed new light when combined with the new ideas of this work.

\section{ACKNOWLEDGMENTS}

This work is supported in part by the National Science Foundation under grant No. PHY1713857 and the Joint Institute for Nuclear Astrophysics (JINA-CEE, www.jinaweb.org), 
NSF-PFC under grant No. PHY-1430152. Support from the faculty research support program at the University of Notre Dame is also acknowledged.

[1] Edward Kolb and Michael Turner, The Early Universe, Frontiers in Physics, Vol. 69 (AddisonWesley Publishing Company, Reading, MA, 1990).

[2] SNO Collaboration, Q. R. Ahmad, R. C. Allen, T. C. Andersen, J. D. Anglin, G. Bühler, J. C. Barton, E. W. Beier, M. Bercovitch, J. Bigu, and others, "Measurement of the Rate of nu_e $+\mathrm{d}->\mathrm{p}+\mathrm{p}+\mathrm{e}-$ Interactions Produced by 8B Solar Neutrinos at the Sudbury Neutrino Observatory," Phys. Rev. Lett. 87, 071301 (2001).

[3] Super-Kamiokande Collaboration, Y. Fukuda, T. Hayakawa, E. Ichihara, K. Inoue, K. Ishihara, H. Ishino, Y. Itow, T. Kajita, J. Kameda, and others, "Evidence for Oscillation of Atmospheric Neutrinos," Phys. Rev. Lett. 81, 1562-1567 (1998).

[4] Steven Weinberg, The Quantum Theory of Fields (Cambridge University Press, Cambridge, England, 1995).

[5] Yoichiro Nambu, "BCS mechanism, quasi supersymmetry, and fermion masses," in New Theories in Physics (World Scientific, Singapore, Kazimierz, Poland, 1988) pp. 1-10; "Quasisupersymmetry, Bootstrap Symmetry Breaking And Fermion Masses," in New Trends in Strong Coupling Gauge Theories (World Scientific, Singapore, Nagoya, Japan, 1988) pp. 3-11.

[6] Wanpeng Tan, "Dark energy and spontaneous mirror symmetry breaking," (2019), arXiv:1908.11838 [gr-qc, physics:hep-ph, physics:hep-th].

[7] T. D. Lee and C. N. Yang, "Question of Parity Conservation in Weak Interactions," Phys. Rev. 104, 254-258 (1956).

[8] I. Yu Kobzarev, L. B. Okun, and I. Ya Pomeranchuk, "On the possibility of experimental observation of mirror particles," Sov J Nucl Phys 3, 837-841 (1966).

[9] S. I. Blinnikov and M. Y. Khlopov, "Possible Astronomical Effects of Mirror Particles," Sov. Astron. 27, 371-375 (1983).

[10] Edward W. Kolb, David Seckel, and Michael S. Turner, "The shadow world of superstring theories," Nature 314, 415 (1985).

[11] Hardy M. Hodges, "Mirror baryons as the dark matter," Phys. Rev. D 47, 456-459 (1993).

[12] Zurab Berezhiani and Luís Bento, "Neutron-Mirror-Neutron Oscillations: How Fast Might 
They Be?" Phys. Rev. Lett. 96, 081801 (2006).

[13] Jian-Wei Cui, Hong-Jian He, Lan-Chun Lü, and Fu-Rong Yin, "Spontaneous mirror parity violation, common origin of matter and dark matter, and the LHC signatures," Phys. Rev. D 85, $096003(2012)$.

[14] R. Foot, "Mirror dark matter: Cosmology, galaxy structure and direct detection," Int. J. Mod. Phys. A 29, 1430013 (2014).

[15] Wanpeng Tan, "Neutron oscillations for solving neutron lifetime and dark matter puzzles," Phys. Lett. B 797, 134921 (2019), arXiv:1902.01837.

[16] Wanpeng Tan, "Neutron-mirror neutron oscillations in stars," (2019), arXiv:1902.03685 [astroph, physics:hep-th, physics:nucl-ex].

[17] Wanpeng Tan, "Neutron-mirror neutron oscillations for solving the puzzles of ultrahigh-energy cosmic rays," (2019), arXiv:1903.07474 [astro-ph, physics:hep-ph].

[18] Wanpeng Tan, "Kaon oscillations and baryon asymmetry of the universe," Phys. Rev. D 100, 063537 (2019), arXiv:1904.03835.

[19] Wanpeng Tan, "Laboratory tests of the ordinary-mirror particle oscillations and the extended CKM matrix," (2019), arXiv:1906.10262 [hep-ex, physics:hep-ph, physics:nucl-ex].

[20] Wanpeng Tan, "Invisible decays of neutral hadrons," (2020), arXiv:2006.10746 [hep-ph].

[21] Georges Obied, Hirosi Ooguri, Lev Spodyneiko, and Cumrun Vafa, "De Sitter Space and the Swampland," (2018), arXiv:1806.08362 [hep-th].

[22] Daniel Harlow and Hirosi Ooguri, "Constraints on Symmetries from Holography," Phys. Rev. Lett. 122, 191601 (2019).

[23] Y. Nambu and G. Jona-Lasinio, "Dynamical Model of Elementary Particles Based on an Analogy with Superconductivity. I," Phys. Rev. 122, 345-358 (1961).

[24] Tohru Eguchi, "New approach to collective phenomena in superconductivity models," Phys. Rev. D 14, 2755-2763 (1976).

[25] G. 't Hooft, "Symmetry Breaking through Bell-Jackiw Anomalies," Phys. Rev. Lett. 37, 8-11 (1976); "Computation of the quantum effects due to a four-dimensional pseudoparticle," Phys. Rev. D 14, 3432-3450 (1976).

[26] F. R. Klinkhamer and N. S. Manton, "A saddle-point solution in the Weinberg-Salam theory," Phys. Rev. D 30, 2212-2220 (1984); F. R. Klinkhamer and P. Nagel, "SU(3) sphaleron: Numerical solution," Phys. Rev. D 96, 016006 (2017). 
[27] Joseph Polchinski, String Theory: Volume 2, Superstring Theory and Beyond (Cambridge University Press, 1998).

[28] Kentaro Hori and Cumrun Vafa, "Mirror Symmetry," (2000), arXiv:hep-th/0002222.

[29] Jacques Colin, Roya Mohayaee, Mohamed Rameez, and Subir Sarkar, "Evidence for anisotropy of cosmic acceleration," Astron. Astrophys. 631, L13 (2019).

[30] Wanpeng Tan, From Neutron and Quark Stars to Black Holes, Preprint: https://osf.io/2jywx (Open Science Framework, 2020). 